\title{
Specialisation in medicine - alpha dog or colony ant?
}

Immanuel Kant was a prime proponent of the idea of specialisation, suggesting that each of us should confine ourselves 'to a certain kind of work distinct from others ... so as to be able to perform it with greater facility and in the greatest perfection'. This drive to be top dog however, is challenged by a school of thought, articulated by author-philosopher Robert Pirsig, that suggests that specialisation is a form of cautious obedience. The thesis goes that the range of human knowledge today is so great that we're all specialists, and this is nowhere more true than in healthcare. In such a world the disconnect between specialisations is so great that one foregoes familiarity with both other colleagues and their area of knowledge. Thus, subspecialising becomes a form of hesitancy, where spontaneity has given way to an obedient narrow focus and no regard to the wider picture.

Clinical Medicine remains a journal targeted to assisting the physician in their day-to-day practice. In an era of increasing specialisation, it has an especial opportunity to do this by keeping the non-specialist updated on the advances in subspecialty areas. It is a pleasure therefore to publish a review of coronary physiology by Ali and colleagues from Leeds. ${ }^{1}$ Symptoms related to coronary artery disease remain a common presentation both in acute care and outpatients. A knowledge of the nuances of revascularisation will assist the generalist who has a patient in whom symptoms don't respond to pharmacological therapy. The article provides an elegant update for non-cardiologists and non-invasive cardiologists of this complex field. The details of the competing technologies are abridged and a practical summary of the approach taken is presented in an excellently illustrated manuscript.

This theme of information for the general physician is showcased in the CME topic of the edition, diabetes. There are state of the art reviews of management of this condition affecting almost one in 10 individuals worldwide, both in the acute setting of ketoacidosis as well as in pregnancy. The first kidney transplant was undertaken over 60 years ago, and there are now over 100,000 solid organ transplants undertaken per year globally. ${ }^{2}$ Managing new onset and chronic diabetes in this increasingly common setting is also reviewed for the generalist reader.
The information technology landscape in healthcare is rapidly evolving, and this coincides with increasing concerns about patient safety, and a drive to patient-engaged shared care. Fritz and colleagues explore doctor and patient attitudes towards sharing medical records in the acute medical setting. ${ }^{3}$ The study shows that there is consensus among both groups about the benefit of a shared summary of the record in the 'real time' setting of an acute illness. Given the robust response rate from patients, firmer conclusions can be drawn from that cohort, and they make for rather dispiriting reading. It would appear that few patients are even aware of the diagnosis for their admission, and generally feel disinclined to ask for clarification. Do read the article for the detail of this dissatisfaction, and engage in the discussion about whether such real-time records access may have positive or negative consequences on trust and quality of care.

Finally, a quick note about the Clinical Medicine website. An updated platform will soon be launched, featuring both manuscripts in print and ahead of publication. In addition to promoting some discussion around the papers in the print journals, and allow for more images and multimedia content, the essence is to make the journal a more interactive offering beyond the onetwo of publication-and-reading. To help host this, we are looking to bring in an associate editor (at a training grade, probably) who will work with the publication team to curate this content: please do look out for this advertisement.

\section{References}

1 Ali N, Patel PA, Malkin C]. Assessment of coronary physiology - the evidence and implications. Clin Med 2019;19:364-8.

2 World Health Organization. Transplantation. WHO. www.who.int/ transplantation/gkt/statistics/en

3 Fritz Z, Schlindwein A, Slowther A-M. Patient engagement or information overload: patient and physician views on sharing the medical record in the acute setting. Clin Med 2019;19:386-91.

Anton Emmanuel Editor-in-chief

\begin{tabular}{|c|c|c|c|}
\hline Michael Almond & Anton Emmanuel & Yash Mahida & Roby Rakhit \\
\hline Cono Ariti & Kate Evans & Nick Manning-Cork & Avan Sayer \\
\hline Paul Belcher & Johanna Feary & Chris Marguerie & Philip Smith \\
\hline Rodger Charlton & Maggie Hammersley & Martin McKee & Angela Star \\
\hline Tim Chevassut & Dylan Harris & Andrew Medford & Cameron Swift \\
\hline Tahseen Chowdhury & Tevfik Ismail & Rahul Mukherjee & Rhys Thomas \\
\hline Elaine Dennison & Vikas Kapil & Mehool Patel & \\
\hline Albert Edwards & Alexandra Lake & Gerrard Phillips & \\
\hline
\end{tabular}

